\title{
Intake of Antioxidants in Relation to Infertility Treatment Outcomes with Assisted Reproductive Technologies
}

\author{
Ming-Chieh $\mathbf{L i}^{1,2}$, Feiby L. Nassan ${ }^{1,3}$, Yu-Han Chiu ${ }^{1}$, Lidia Mínguez-Alarcón ${ }^{3}$, Paige L. \\ Williams $^{4,5}$, Irene Souter ${ }^{6}$, Russ Hauser ${ }^{3,4,6}$, and Jorge E. Chavarro ${ }^{1,4,7}$ on behalf of EARTH \\ Study team \\ 1.Department of Nutrition, Harvard T.H. Chan School of Public Health, Boston, MA 02115, USA \\ 2.Department of Public Health, China Medical University College of Public Health, Taichung \\ 40402, Taiwan \\ 3.Department of Environmental Health, Harvard T.H. Chan School of Public Health, Boston, MA \\ 02115, USA \\ 4.Department of Epidemiology, Harvard T.H. Chan School of Public Health, Boston, MA 02115, \\ USA \\ 5.Department of Biostatistics, Harvard T.H. Chan School of Public Health, Boston, MA 02115, \\ USA \\ 6. Department of Obstetrics and Gynecology, Division of Reproductive Endocrinology and \\ Infertility, Massachusetts General Hospital Fertility Center, Harvard Medical School, Boston, MA \\ 02114, USA \\ 7.Channing Division of Network Medicine, Brigham and Women's Hospital and Harvard Medical \\ School, Boston, MA 02115, USA.
}

\section{Abstract}

Background: Randomized trials of supplementation with antioxidant mixtures during infertility treatment show no benefit on pregnancy or live birth rate. However, the roles of individual antioxidants are poorly understood. We examined the association of baseline intake of vitamins A, $\mathrm{C}, \mathrm{E}$, and carotenoids with outcomes of assisted reproductive technologies (ART).

Methods: We followed 349 women undergoing a total of 588 ART cycles for infertility treatment at the Massachusetts General Hospital. We assessed antioxidant intakes from food and supplements before treatment using a validated food frequency questionnaire. We used generalized linear mixed models to account for multiple ART cycles per woman while adjusting for confounding.

Corresponding author: Dr. Jorge E. Chavarro., Department of Nutrition, Harvard School of Public Health, 665 Huntington Ave., Boston, MA 02115; Phone: 617-432-4584; jchavarr@hsph.harvard.edu.

Conflicts of interest: The authors declare that there is no conflict of interest.

Availability of data and code for replication: All data used and computing code developed for the analyses described in this article is available upon request, subject to the access requirements of the institutions. 
Results: Mean (SD) age and body mass index were 35.1 (4.0) years and $24.1(4.3) \mathrm{kg} / \mathrm{m}^{2}$. Total intake of vitamins A, C, and E were not associated with the probability of live birth. Women in the highest intake category of $\beta$-carotene from foods had a lower probability of live birth than women in the lowest intake quartile ( $50 \%$ vs $22 \%$; p-trend $=0.03$ ); for lutein and zeaxanthin, the probability for the highest intake group was $44 \%$ vs $28 \%$ for the lowest). Intake of $\beta$-carotene from supplements and intakes of retinol and all other carotenoids was unrelated to live birth rates.

Conclusions: We found unexpected inverse associations of $\beta$-carotene intake from foods and of lutein and zeaxanthin intake with live birth rates. Within the observed intake ranges, total consumption of vitamins A, C, and E prior to starting infertility treatment with ART were not associated with live birth rates.

\section{Keywords}

infertility; in vitro fertilization; probability of live birth; assisted reproductive technology; antioxidants; vitamin A; vitamin C; vitamin E; carotenoids

\section{Introduction}

Infertility is a global public health concern. An estimated 48.5 million couples worldwide are infertile, ${ }^{1}$ and approximately $40 \%-50 \%$ percent of cases are attributed to female factors. 2,3 Assisted reproductive technologies (ART) are a cornerstone of infertility treatment, with more than 600,000 cycles performed each year in North America and Europe alone. ${ }^{4-6}$ Nevertheless, success rates remain relatively low; in 2015 in the US, approximately only one of three ART cycles started with the intention to transfer an embryo resulted in a live birth. ${ }^{4}$ It is thus of vital importance to identify potentially modifiable predictors of treatment outcome, including diet. $^{7}$

Growing evidence indicates that oxidative stress impairs gamete and embryo development during ART. ${ }^{8}$ In addition, infertile women have been reported to have a lower total antioxidant status. ${ }^{9,10}$ It has thus been suggested that antioxidant supplements may improve infertility treatment outcomes with ART. ${ }^{11}$ However, high intake of some vitamins, such as vitamin A, may result in adverse reproductive outcomes. ${ }^{12,13}$ Furthermore, the relation between micronutrients and ART outcomes may not necessarily be linear. ${ }^{14}$

While several randomized controlled trials (RCTs) have addressed this question, their findings are inconsistent. A 2017 Cochrane review of RCTs concluded that antioxidant supplementation during the course of infertility treatment showed limited benefit for increasing the probability of pregnancy or live birth and that, among women undergoing ART specifically, antioxidants were not associated with an increased live birth rate. ${ }^{11}$ However, as acknowledged by the authors, this evidence suffers from multiple important gaps. First, few trials reported effects of the intervention on live birth rates. In addition, few of the trials included in the meta-analysis tested the same intervention and estimated pooled data from interventions as dissimilar as multiple micronutrient blends (including proprietary blends whose composition cannot be established) to selected combinations of specific micronutrients at considerably different doses, to interventions of substances without direct antioxidant potential. These issues make it difficult to draw strong conclusions regarding the 
effects-or lack thereof-of antioxidants on ART outcomes. A particularly important gap to move the field forward is to identify which specific antioxidants and at which doses may offer benefit to inform the design of new trials. To advance this goal, we conducted a prospective study to examine the association between baseline intake of specific micronutrients with direct antioxidant capacity (vitamins A, C, and E; retinol; and carotenoids) from food and supplemental sources and outcomes of infertility treatment with ART.

\section{Methods}

\section{Study population}

Study participants were women enrolled in the Environment and Reproductive Health (EARTH) Study, an ongoing prospective cohort started in 2004 to explore environmental and lifestyle determinants of fertility among couples presenting for evaluation or treatment of infertility to the Massachusetts General Hospital (MGH) Fertility Center (Boston, MA). ${ }^{15}$ Women were eligible to participate if they were between 18 and 45 years and planned to use their own gametes for infertility treatment. Women whose treating physician later determined that using donor eggs was clinically necessary remained in the study. Among women referred by physicians, approximately $60 \%$ of those approached by the research nurses enrolled in the study. Diet assessment was introduced to the study in 2007.

At study entry, study nurses measured participants' height and weight and administered a brief questionnaire aimed at collecting basic demographic, medical, and lifestyle information. Participants were then asked to complete a longer questionnaire at home, including a food frequency questionnaire (FFQ). The current analysis includes all women who completed a FFQ and subsequently underwent at least one autologous ART cycle by November 2016 ( $\mathrm{N}=349$ women; 588 ART cycles). Oocyte donation cycles ( 25 cycles) were excluded from the analysis). The study was approved by the institutional review boards of the MGH and the Harvard T.H. Chan School of Public Health. All participants provided written informed consent after the study procedures were explained by a research nurse.

\section{Dietary assessment}

Diet was assessed at baseline using an extensively validated FFQ. ${ }^{16,17}$ In the validation study, the correlation between FFQ-assessed carotenoid intake and plasma levels of the corresponding carotenoid ranged between 0.59 and $0.81 .{ }^{16}$ Similar results were obtained for vitamins $\mathrm{A}, \mathrm{C}$, and $\mathrm{E}$, and carotenoid when FFQ-assessments were compared to prospectively collected diet records. ${ }^{17}$ Vitamin A, expressed as mcg/day of retinol activity equivalents, includes intake of retinol and of provitamin A carotenoids weighed by their retinol forming capacity. Participants reported how often they consumed specified amount of 131 food items during the previous year. Nutrient intakes were estimated by summing the nutrient contribution of each food and supplement included in the questionnaire, taking into consideration the brands of specific supplements and breakfast cereals. Nutrient contents for each item in the questionnaire were obtained from the US Department of Agriculture with supplemental data obtained from food manufacturers. ${ }^{18}$ Nutrient intakes were energy adjusted using the residual method. ${ }^{19}$ Principal components analysis (PCA) was used to 
derive food patterns based on 40 pre-defined food groups. ${ }^{20}$ The 'Prudent" pattern was characterized by high intakes of fish, fruits, cruciferous vegetables, yellow vegetables, tomatoes, leafy green vegetables and legumes; The "Western" pattern was characterized by high intakes of processed meat, full-fat dairy, fries, refined grains, pizza and mayonnaise. All participants were given a score for each pattern according to their adherence to these patterns. The scores ranged from -2.4 to 5.0 for Prudent" pattern and -1.9 to 3.7 for "Western" pattern. A higher pattern score can be interpreted as higher adherence to the pattern.

\section{Clinical management and assessment of outcomes}

Clinical information was abstracted by trained study staff from the patients' electronic medical records. Details of patient clinical management was previously described elsewhere.

${ }^{21}$ Briefly, after completing a cycle of oral contraceptives, participants underwent one of three stimulation protocols as clinically indicated: 1) luteal-phase gonadotropin-releasing hormone (GnRH) agonist protocol, 2) follicular-phase GnRH-agonist/ flare protocol; or 3) follicular-phase GnRH-antagonist protocol. Clinical staff monitored patients during gonadotropin stimulation for serum estradiol $\left(\mathrm{E}_{2}\right)$, follicle size and counts, and endometrial thickness, and administered human chorionic gonadotropin (hCG) approximately 35-36 hours before the scheduled oocyte retrieval to induce oocyte maturation. Oocytes were classified by embryologists as germinal vesicle, metaphase I, metaphase II, or degenerated. Embryologists then determined fertilization rate as the number of oocytes with 2 pronuclei divided by the number of metaphase II oocytes at 17 to 20 hours after either in vitro fertilization or intracytoplasmic sperm injection. Early ART end points referred to those preceding embryo transfer, including markers of ovarian responses to stimulation (peak $\mathrm{E}_{2}$ levels, endometrial thickness, metaphase II and total oocytes), and fertilization rate. Patients undergoing cryo-thaw cycles underwent endometrial preparation protocols as clinically indicated. Following embryo transfer, all clinical outcomes (i.e. implantation, clinical pregnancy, and live birth) were assessed identically for fresh and cryo-thaw cycles. We defined implantation as a serum $\beta$-hCG level $>6 \mathrm{mIU} / \mathrm{mL}$, measured approximately 17 days after oocyte retrieval, clinical pregnancy as the presence of intrauterine gestational sac(s) on transvaginal ultrasonography at 6 weeks of gestation, and live birth as the birth of a neonate on or after 24 weeks of gestation. The denominator for all clinical outcomes was the total number of ART cycles started.

\section{Statistical analysis}

We divided participants into quartiles of intake for each antioxidant. Antioxidant intakes from supplements and food were analyzed separately and jointly. The association of baseline demographic, dietary and reproductive characteristics with intake antioxidants was evaluated using Kruskal-Wallis tests for continuous variables and Chi-square tests for categorical variables. Generalized linear mixed models with random intercepts were used to evaluate the associations of intakes of vitamin A, C, and E and carotenoid intake with ART outcomes. A normal distribution and identity link were specified for continuous outcomes (peak $\mathrm{E}_{2}$ levels, endometrial thickness); a Poisson distribution and log link function for count outcomes (number of total and mature oocytes retrieved); and a binomial distribution and a logit link function for proportions (fertilization and clinical outcomes). Population marginal means 
were used to present population averages adjusted for the covariates (at the mean level for continuous variables and for categorical variables at a value weighted according to their frequencies) in the model. ${ }^{22}$ Tests for linear trend across quartiles of intake were conducted using the median nutrient intake in each quartile as a continuous variable in the regression models. Confounding was evaluated using previous knowledge and taking into consideration the associations between intake of antioxidants with baseline characteristics. ${ }^{4,14,21,23,24}$ Multivariable adjusted models were included simultaneously terms for the other antioxidants, age, BMI, smoking, total calorie intake, dietary patterns, intakes of folate and vitamin B12, and treatment protocol. We tested whether the association between intake of antioxidants and the probability of live birth was modified by age ( $\geq 35 \mathrm{vs}$. $<35$ ), BMI ( $\geq 25$ vs. $<25$ ) and smoking status (ever vs. never) by introducing cross product terms to the multivariable adjusted models. When there was a suggestive interaction effect (p-value $<0.10)$, stratified analysis was further conducted to investigate whether the changes in probabilities of live birth varied by age, smoking status, and BMI. Primary analyses included all autologous cycles for a woman, including fresh and cryo-thaw cycles. We performed sensitivity analyses to test the robustness of the findings and modeling assumptions, which included modeling intake as a continuous linear term, as a restricted cubic spline and using different distribution-based groupings (tertiles, quintiles). We also performed analyses coadjusting for male partner intake of the same micronutrients among couples where male partner diet data was available ( $\mathrm{N}=170$ women, 301 cycles) and restricting analysis to fresh cycles only ( $\mathrm{n}=338$ women, 503 cycles). We performed all statistical analyses using SAS 9.4 (SAS Institute, Cary, NC, USA).

\section{Results}

The analysis included 349 women who collectively underwent 588 ART cycles of which 233 (40\%) resulted in a live birth. Mean (standard deviation) age was 35.1 (4.0) years and BMI was $24.1(4.3) \mathrm{kg} / \mathrm{m}^{2}$ (Table 1). The majority of women were white $(83 \%)$ and had a college degree $(98 \%)$. Less than a third of women (26\%) had ever smoked. The most common initial infertility diagnosis was unexplained (41\%), followed by male factor (32\%). Approximately one third of women (38\%) reported at least one prior pregnancy. Vitamin A, C, E, and carotenoid intakes were positively related to each other, to intakes of folate and B12, and to dietary patterns, but not to alcohol and caffeine consumption or personal characteristics (age, BMI, smoking status, race, and education) (Table 1; Supplemental eTable 1). The median time between diet assessment and the start the first ART cycle was 133 days.

Total intakes of vitamins $\mathrm{C}$ and $\mathrm{E}$ were not associated with intermediate outcomes, including endometrial thickness, peak $\mathrm{E}_{2}$ levels, oocyte yield and fertilization rates (Supplemental eTable 3). Vitamin A total intake was positively related to endometrial thickness. The adjusted mean endometrial thicknesses in $\mathrm{mm}(95 \% \mathrm{CI})$ for women in the lowest quartile of vitamin A total intake was $10.6(10.0-11.2)$ and in the highest 9.8 (9.2-10.3) (p-trend=0.02). When we considered vitamin A intake from supplements separately from food intake, vitamin A from supplements was inversely related to total and M2 oocyte yield, while vitamin A from foods was unrelated to this outcome (Supplemental eTable 3). The adjusted mean number of $\mathrm{M} 2$ oocytes $(95 \% \mathrm{CI})$ for women in the lowest quartile of vitamin A intake from supplements was $10.6(8.9-12.7)$ and $7.9(7.0-9.0)$ in the highest (p-trend=0.04); The 
adjusted mean number of total oocytes were $12.9(10.9-15.3)$ in the lowest quartile and 9.2 (8.2-10.4) in the highest ( $\mathrm{p}$-trend=0.01). When we considered vitamin $\mathrm{E}$ intake from supplements separately from food intake, vitamin E from supplements was positively related to total oocyte yield. The adjusted mean number of total oocytes $(95 \% \mathrm{CI})$ for women in the lowest quartile of vitamin $\mathrm{E}$ intake from supplements was 8.7 (7.4-10.3) and in the highest $11.0(9.7-12.4)$ (p-trend=0.05), while vitamin $\mathrm{E}$ from foods was unrelated to this outcome (Supplemental eTable 3).

Intakes of vitamin $\mathrm{A}, \mathrm{C}$, and $\mathrm{E}$ were not related to the probability of implantation, clinical pregnancy or live birth per initiated treatment cycle (Table 2). The adjusted probability of live birth $(95 \% \mathrm{CI})$ for women in the lowest quartile intake categories was $25 \%(17 \%-35 \%)$ and in the highest $31 \%(23 \%-42 \%)$ for vitamin A total intake; $30 \%(21 \%-40 \%)$ for the lowest and 32\% (24\%-42\%) for the highest vitamin C total intake; and 38\% (27\%-50\%) for the lowest and 29\% (21\%-39\%) for the highest vitamin E total intake (Table 2). Results did not change when intakes from foods and supplements were considered separately (Table 2).

We then evaluated the relationship between intake of retinol and individual carotenoids with intermediate ART outcomes (Table 3 and supplemental eTable 4). Intake of a-carotene was positively related to endometrial thickness. The adjusted mean endometrial thicknesses in $\mathrm{mm}(95 \% \mathrm{CI})$ for women in the lowest quartile of a-carotene total intake were 9.8 (9.2$10.4)$ and in the highest $10.6(10.0-11.2)$ ( $\mathrm{p}$-trend=0.05). No other associations were observed between carotenoids and intermediate outcomes (Supplemental eTable 4).

Intake of retinol and most carotenoids were unrelated to the probabilities of implantation, clinical pregnancy or live birth (Table 3). The adjusted probability of live birth (95\% CI) for was $25 \%(17 \%-35 \%)$ for the lowest quartile of total intake of retinol and 37\% (27\%-47\%) for the highest; $33 \%(24 \%-43 \%)$ for the lowest quartile of a-carotene and 35\% (25\%-46\%) the highest; $36 \%(25 \%-48 \%)$ the lowest quartile of $\beta$-carotene and $32 \%(21 \%-44 \%)$ for the highest; $33 \%(25 \%-42 \%)$ for the lowest quartile of $\beta$-cryptoxanthin and 33\% (24\%-43\%) for the highest; and $34 \%(26 \%-43 \%)$ for the lowest quartile of lycopene and $36 \%(27 \%$ $-45 \%)$ for the highest. When we considered $\beta$-carotene intake from food and supplemental sources eparately, higher intake from foods was related to lower live birth rates (Table 3). The adjusted probability of live birth $(95 \% \mathrm{CI})$ for women in the lowest and highest intake categories were 50\% (36-64\%) and 22\% (13-35\%) for $\beta$-carotene from foods ( $\mathrm{p}$, trend=0.03). A similar inverse relationship was present for intake of lutein and zeaxanthin. The adjusted probability of live birth $(95 \% \mathrm{CI})$ for women in the lowest category of lutein and zeaxanthin was $44 \%(33 \%-56 \%)$ and for the highest was $28 \%(19 \%-38 \%)$, respectively. Adjustment for pesticide residue contamination based on USDA surveillance data ${ }^{25,26}$ did not change the results. When we evaluated the association between the main food contributors of $\beta$-carotene and lutein and zeaxanthin intake in this population, only intake of sweet potatoes was related to live birth rate (eTable 5). $\beta$-carotene intake from supplements was unrelated to these outcomes (Table 3). The adjusted probability of live birth $(95 \%$ CI) for women in bottom and top intake categories were $32 \%(24 \%-42 \%)$ and $29 \%(20 \%-39 \%)$ (Table 3). 
We found no evidence that smoking, age, or BMI modified the relation between intake of antioxidants and outcomes of infertility treatment.

We performed sensitivity analyses aimed at evaluating the robustness of our findings and assumptions. Univariate associations differed little from multivariable-adjusted estimates (eTable 6). None of the nutrients evaluated, including $\beta$-carotene from foods and lutein and zeaxanthin, was associated to the probability of live birth when intake was modeled as a continuous linear term or when modeled as a restricted cubic spline to allow for nonlinearity. Moreover, the associations for intake of $\beta$-carotene from foods and lutein and zeaxanthin with the probability of live birth were highly sensitive to the choice of cutoffs, with large changes in magnitude associated with minor changes in cutoff values. Findings did not change when co-adjusting for male partner intake of the same micronutrients in the subgroup of women where these data were available ( $\mathrm{N}=170$ women, 301 cycles), or when restricted to fresh cycles ( $\mathrm{N}=338$ women, 503 cycles).

\section{Discussion}

In this prospective cohort of women undergoing infertility treatment with ART, we identified a positive relationship of vitamin $\mathrm{E}$ intake from supplements, and a negative one of vitamin A from supplements, with oocyte yield. While these relationships did not translate into differences in clinically relevant outcomes, they may be of interest in settings where the goal is improving oocyte yields, such as egg donation programs. When intake of vitamin A was separated into intake from retinol and intake of provitamin A carotenoids, we found an inverse association between $\beta$-carotene intake from food sources with live birth rates and a similar relationship with intake of lutein and zeaxanthin. This pattern, coupled with the lack of associations with total vitamin A, $\beta$-carotene from supplement, total $\beta$-carotene and all other carotenoids intake suggests that these associations may not be due to the nutrients themselves but may instead reflect either chance findings or associations with primary food sources. Overall, our findings suggest that habitual intake of antioxidants from foods and supplements prior to infertility treatment offers no benefit on ART outcomes.

There is an extensive literature on the relation between antioxidants and outcomes of infertility treatments, including several randomized clinical trials addressing this question. Of note, a Cochrane systematic review and meta-analysis of these RCTs concluded that the current evidence provides little support for supplementing women undergoing infertility treatment with antioxidants. ${ }^{11}$ Importantly, this review also highlighted many of the limitations of the current literature including the heterogeneity of interventions, which make it difficult to attribute any specific observed effects to a specific antioxidant or dose. ${ }^{11}$ Most of the literature in this field has investigated the effect of supplementation with mixtures of antioxidants or other supplements including antioxidants, but epidemiologic studies and RCTs focusing on single antioxidants, including vitamins A, C, and E, or carotenoids, are relatively limited, and findings are inconsistent. ${ }^{27-29}$ For example, Ruder and colleagues found that vitamin $\mathrm{C}, \beta$-carotene, and vitamin $\mathrm{E}$ were related to better ART outcomes in subgroups of women defined by BMI or age. ${ }^{27}$ Similarly, Crha and collaborators found that vitamin $\mathrm{C}$ supplementation increased pregnancy rate primarily among non-smokers. ${ }^{28} \mathrm{We}$ found no evidence of effect modification by age, BMI, or smoking status in our study. 
Instead, our findings are better aligned with the overall conclusion of the Cochrane review on women undergoing in vitro fertilization or intracytoplasmic sperm injection, ${ }^{11}$ as well as with the findings of previous RCTs focusing specifically on supplementation with these nutrients, alone or in combination of no benefit of antioxidants in the setting of assisted reproduction. ${ }^{30-33}$

In our study, $\beta$-carotene intake from food sources, but not from supplements, was inversely related to the probability of live birth. We found a similar inverse relation between intake of lutein and zeaxanthin and the probability of live birth. It is important to briefly review the basic differences and similarities of retinol, provitamin A carotenoids, and non-provitamin A carotenoids in discussing these findings. The term vitamin A refers to both preformed vitamin A (retinol and its esters) and provitamin A carotenoids (largely $a$-carotene, $\beta$ carotene, and $\beta$-cryptoxanthin). Retinol can be reversibly oxidized to retinal, which exhibits all of the biologic activities of retinol; or further oxidized to retinoic acid, which is the primary active metabolite of vitamin A. ${ }^{34}$ Provitamin A carotenoids can be endogenously converted into retinoic acid whereas other carotenoids, such as lutein, zeaxanthin, and lycopene, are not precursors of vitamin A but do have direct antioxidant capacity. Seventyfive percent of $\beta$-carotene intake in this study population is accounted for by five foods: lettuce, spinach, carrots, sweet potatoes, and kale. Three of these five foods (lettuce, spinach, and kale) account for $71 \%$ of lutein and zeaxanthin intake. These five foods are known to have modest pesticide residue contamination in the US food supply. ${ }^{35}$ We have previously reported that intake of fruits and vegetables with high levels of contamination with pesticide residues is related to lower ART success. ${ }^{36}$ However, adjustment for pesticide residues did not change the results. Furthermore, the only parent food related to live births was sweet potato, which accounts for $6.8 \%$ of $\beta$-carotene intake and is not an important source of lutein and zeaxanthin in this population. It is also possible that some of these relations represent true associations. In support of this interpretation, Ruder and colleagues reported that higher intake of $\beta$-carotene was related to longer time to pregnancy among women over 35 years (the median age in our population) who participated in a RCT of infertility treatment, although the opposite relation was observed among younger women. ${ }^{27}$ We did not observe evidence of effect modification by age in this study. A third, and highly likely, possible interpretation is that these associations are a chance finding. The observed pattern of an inverse relation with intake from food sources but no relation with total intake or intake from supplements and no relation with retinol or the other carotenoids supports this third interpretation. Furthermore, the fact that these associations were highly sensitive to modeling assumptions, including major changes in magnitude when modeled using different distribution based cutoffs and when modeled using restricted cubic splines, decreases our confidence that these findings may represent true biological associations. Nevertheless, given the relative scarcity of data regarding the relation of retinol and carotenoids with fertility and infertility treatment outcomes, more studies are needed to confirm or refute these findings.

Strengths of our study include its prospective design and complete participant follow-up, and thorough assessment of intermediate and clinical outcomes, all of which aid in the interpretation of the findings. The availability of comprehensive demographic, lifestyle and dietary data also allowed us to account for a broad range of potential confounders including 
highly correlated nutrients. The size of the cohort allowed us to evaluate clinically relevant outcomes with sufficient statistical power, including live birth. Important limitations of the study include non-differential exposure misclassification by virtue of using FFQs for diet assessment and due to differences in timing of diet assessment in relation to treatment initiation. Also, since FFQs are designed to assess habitual diet, recent changes to diet and changes in diet after diet assessment but before treatment would not be adequately captured.

In conclusion, in this large prospective cohort we unexpectedly found an inverse relation of $\beta$-carotene intake from foods, but not from supplements, and of lutein and zeaxanthin, with live birth rates. More importantly, within the observed intake ranges, we found no association between total baseline intakes of vitamins A, C, and E - from food and supplemental sources - of retinol and of all other carotenoids with outcomes of ART-based infertility treatment. These findings are in agreement with the results of a Cochrane systematic review of RCTs which also show no benefit of antioxidant supplementation for females undergoing in vitro fertilization or intracytoplasmic sperm injection, ${ }^{11}$ and, despite biologic plausibility, do not support the hypothesis that female partner's baseline intake of antioxidants plays a major role in determining the outcome of infertility treatment.

\section{Supplementary Material}

Refer to Web version on PubMed Central for supplementary material.

\section{Acknowledgments}

Sources of funding: This work was supported by the National Institutes of Health [P30ES000002, R01ES009718, R01ES022955 and P30DK046200]. Dr. Li was supported by the Ministry of Science and Technology, Taiwan [MOST 106-2917-I-564-066].

\section{References}

1. Mascarenhas MN, Flaxman SR, Boerma T, Vanderpoel S, Stevens GA. National, regional, and global trends in infertility prevalence since 1990: a systematic analysis of 277 health surveys. PLoS Med 2012;9(12):e1001356. [PubMed: 23271957]

2. Boivin J, Bunting L, Collins JA, Nygren KG. International estimates of infertility prevalence and treatment-seeking: potential need and demand for infertility medical care. Hum Reprod 2007;22(6): 1506-12. [PubMed: 17376819]

3. Hart R Unexplained infertility, endometriosis, and fibroids. Bmj 2003;327(7417):721-4. [PubMed: 14512481]

4. Centers for Disease Control and Prevention ASfRM, Society for Assisted Reproductive Technology. 2015 Assisted Reproductive Technology Fertility Clinic Success Rates Report.. Atlanta (GA): US Dept of Health and Human Services 2017.

5. Calhaz-Jorge C, De Geyter C, Kupka MS, de Mouzon J, Erb K, Mocanu E, Motrenko T, Scaravelli G, Wyns C, Goossens V. Assisted reproductive technology in Europe, 2013: results generated from European registers by ESHRE. Hum Reprod 2017;32(10):1957-1973. [PubMed: 29117383]

6. Canadian Fertility and Andrology Society. Assisted Reproductive Technologies (ART) in Canada: 2015 Results from the Canadian ART Register (CARTR). 2016.

7. Gaskins AJ, Chavarro JE. Diet and fertility: a review. Am J Obstet Gynecol 2017.

8. du Plessis SS, Makker K, Desai NR, Agarwal A. Impact of oxidative stress on IVF. Expert review of obstetrics \& gynecology 2008;3(4):539-554. 
9. Polak G, Kozioł-Montewka M, Gogacz M, Błaszkowska I, Kotarski J. Total antioxidant status of peritoneal fluid in infertile women. European Journal of Obstetrics \& Gynecology and Reproductive Biology 2001;94(2):261-263. [PubMed: 11165736]

10. Fenkci V, Fenkci S, Yilmazer M, Serteser M. Decreased total antioxidant status and increased oxidative stress in women with polycystic ovary syndrome may contribute to the risk of cardiovascular disease. Fertility and sterility 2003;80(1):123-127. [PubMed: 12849813]

11. Showell MG, Mackenzie-Proctor R, Jordan V, Hart RJ. Antioxidants for female subfertility. Cochrane Database Syst Rev 2017;7:Cd007807. [PubMed: 28752910]

12. Rothman KJ, Moore LL, Singer MR, Nguyen US, Mannino S, Milunsky A. Teratogenicity of high vitamin A intake. N Engl J Med 1995;333(21):1369-73. [PubMed: 7477116]

13. Cohlan SQ. Excessive intake of vitamin A as a cause of congenital anomalies in the rat. Science 1953;117(3046):535-6.

14. Gaskins AJ, Afeiche MC, Wright DL, Toth TL, Williams PL, Gillman MW, Hauser R, Chavarro JE. Dietary folate and reproductive success among women undergoing assisted reproduction. Obstet Gynecol 2014;124(4):801-9. [PubMed: 25198264]

15. Hauser R, Meeker JD, Duty S, Silva MJ, Calafat AM. Altered semen quality in relation to urinary concentrations of phthalate monoester and oxidative metabolites. Epidemiology 2006;17(6):68291. [PubMed: 17003688]

16. Yuan C, Spiegelman D, Rimm EB, Rosner BA, Stampfer MJ, Barnett JB, Chavarro JE, Rood JC, Harnack LJ, Sampson LK, Willett WC. Relative Validity of Nutrient Intakes Assessed by Questionnaire, 24-Hour Recalls, and Diet Records Compared With Urinary Recovery and Plasma Concentration Biomarkers: Findings for Women. Am J Epidemiol 2017.

17. Yuan C, Spiegelman D, Rimm EB, Rosner BA, Stampfer MJ, Barnett JB, Chavarro JE, Subar AF, Sampson LK, Willett WC. Validity of a Dietary Questionnaire Assessed by Comparison With Multiple Weighed Dietary Records or 24-Hour Recalls. Am J Epidemiol 2017;185(7):570-584. [PubMed: 28338828]

18. United States Department of Agriculture and Agricultural Research Service. USDA National Nutrient Database for Standard Reference, Release 25. https://www.ars.usda.gov/northeast-area/ beltsville-md-bhnrc/beltsville-human-nutrition-research-center/nutrient-data-laboratory/docs/sr25home-page/. Accessed Aug 13, 2018.

19. Willett WC, Howe GR, Kushi LH. Adjustment for total energy intake in epidemiologic studies. Am J Clin Nutr 1997;65(4 Suppl):1220S-1228S; [PubMed: 9094926]

20. Gaskins AJ, Colaci DS, Mendiola J, Swan SH, Chavarro JE. Dietary patterns and semen quality in young men. Hum Reprod 2012;27(10):2899-907. [PubMed: 22888168]

21. Chavarro JE, Ehrlich S, Colaci DS, Wright DL, Toth TL, Petrozza JC, Hauser R. Body mass index and short-term weight change in relation to treatment outcomes in women undergoing assisted reproduction. Fertil Steril 2012;98(1):109-16. [PubMed: 22607889]

22. Searle SR, Speed FM, Milliken GA. Population marginal means in the linear model: an alternative to least squares means. The American Statistician 1980;34(4):216-221.

23. Gaskins AJ, Chiu YH, Williams PL, Ford JB, Toth TL, Hauser R, Chavarro JE. Association between serum folate and vitamin B-12 and outcomes of assisted reproductive technologies. Am J Clin Nutr 2015;102(4):943-50. [PubMed: 26354529]

24. Waylen AL, Metwally M, Jones GL, Wilkinson AJ, Ledger WL. Effects of cigarette smoking upon clinical outcomes of assisted reproduction: a meta-analysis. Hum Reprod Update 2009;15(1):3144. [PubMed: 18927070]

25. Hu Y, Chiu YH, Hauser R, Chavarro J, Sun Q. Overall and class-specific scores of pesticide residues from fruits and vegetables as a tool to rank intake of pesticide residues in United States: A validation study. Environ Int 2016;92-93:294-300.

26. Chiu YH, Williams PL, Minguez-Alarcon L, Gillman M, Sun Q, Ospina M, Calafat AM, Hauser R, Chavarro JE. Comparison of questionnaire-based estimation of pesticide residue intake from fruits and vegetables with urinary concentrations of pesticide biomarkers. J Expo Sci Environ Epidemiol 2018;28(1):31-39. [PubMed: 28930298]

Epidemiology. Author manuscript; available in PMC 2020 May 01. 
27. Ruder EH, Hartman TJ, Reindollar RH, Goldman MB. Female dietary antioxidant intake and time to pregnancy among couples treated for unexplained infertility. Fertil Steril 2014;101(3):759-66. [PubMed: 24355050]

28. Crha I, Hruba D, Ventruba P, Fiala J, Totusek J, Visnova H. Ascorbic acid and infertility treatment. Cent Eur J Public Health 2003;11(2):63-7. [PubMed: 12884545]

29. Kazemi A, Ramezanzadeh F, Nasr-Esfahani MH. The relations between dietary antioxidant vitamins intake and oxidative stress in follicular fluid and ART outcomes. Iran J Reprod Med 2015;13(9):533-40. [PubMed: 26568757]

30. Cicek N, Eryilmaz OG, Sarikaya E, Gulerman C, Genc Y. Vitamin E effect on controlled ovarian stimulation of unexplained infertile women. J Assist Reprod Genet 2012;29(4):325-8. [PubMed: 22302530]

31. Aleyasin A, Aghahosseini M, Mohseni M, Mahdavi A. Effects of pentoxifylline and vitamin E on pregnancy rate in infertile women treated by ZIFT: a randomized clinical trial. International Journal of Reproductive BioMedicine 2009;7(4):175-179.

32. Mier-Cabrera J, Genera-García M, la Jara-Díaz D, Perichart-Perera O, Vadillo-Ortega F, Hernández-Guerrero C. Effect of vitamins $\mathrm{C}$ and $\mathrm{E}$ supplementation on peripheral oxidative stress markers and pregnancy rate in women with endometriosis. International Journal of Gynecology \& Obstetrics 2008;100(3):252-256. [PubMed: 18005966]

33. Youssef MA, Abdelmoty HI, Elashmwi HA, Abduljawad EM, Elghamary N, Magdy A, Mohesen MN, Abdella RM, Bar MA, Gouda HM. Oral antioxidants supplementation for women with unexplained infertility undergoing ICSI/IVF: randomized controlled trial. Human Fertility 2015;18(1):38-42. [PubMed: 24989721]

34. Solomons NW. Vitamin A In: Erdman JWMI, Zeisel SH, ed. Present Knowledge in Nutrition. 10th Edition ed John Wiley \& Sons Ltd, 2012;149-184.

35. US Department of Agriculture. Pesticide Data Program Databases and Annual Summaries.

36. Chiu YH, Williams PL, Gillman MW, Gaskins AJ, Minguez-Alarcon L, Souter I, Toth TL, Ford JB, Hauser R, Chavarro JE. Association Between Pesticide Residue Intake From Consumption of Fruits and Vegetables and Pregnancy Outcomes Among Women Undergoing Infertility Treatment With Assisted Reproductive Technology. JAMA Intern Med 2017. 


\section{을 \\ }
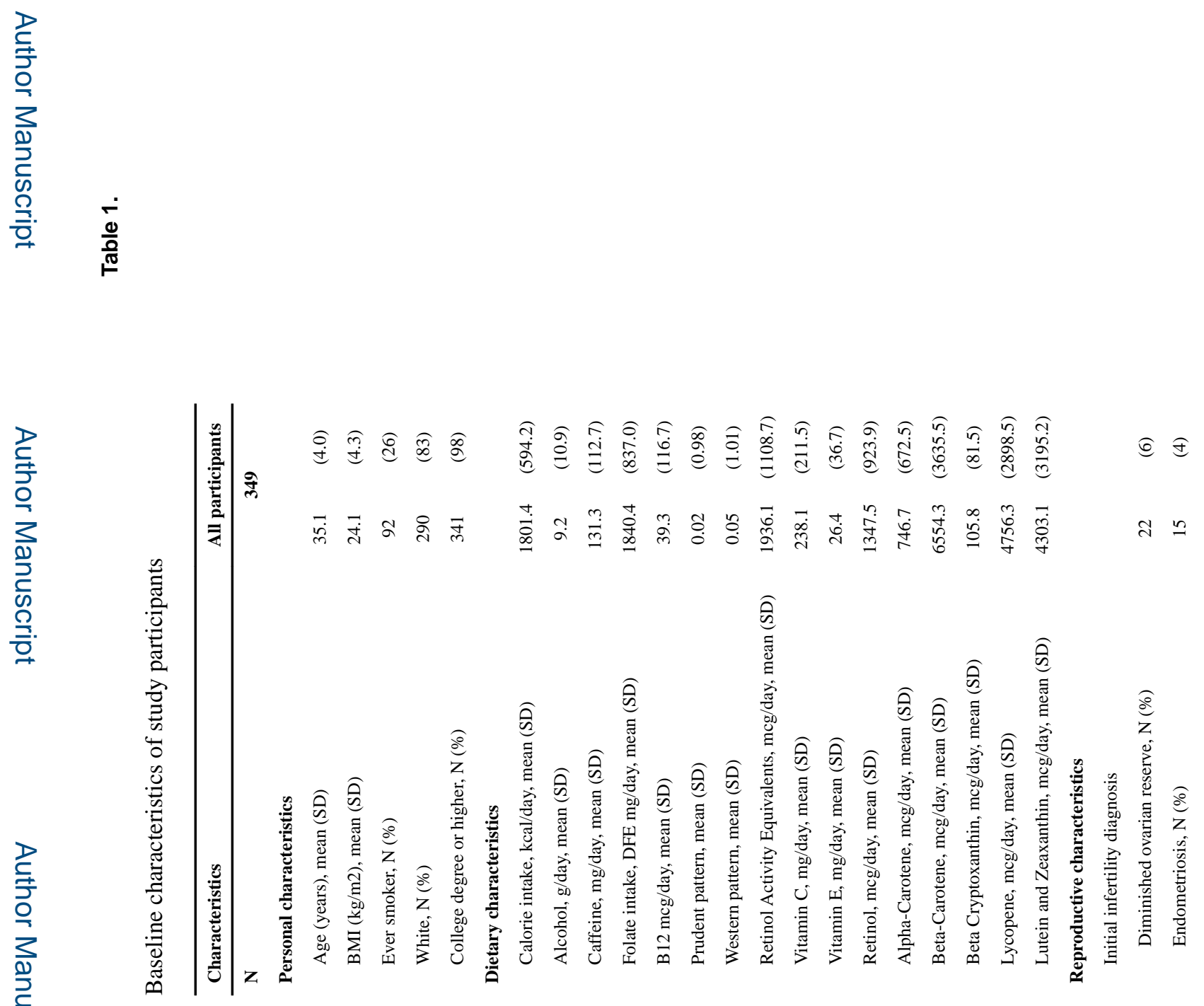

Epidemiology. Author manuscript; available in PMC 2020 May 01. 


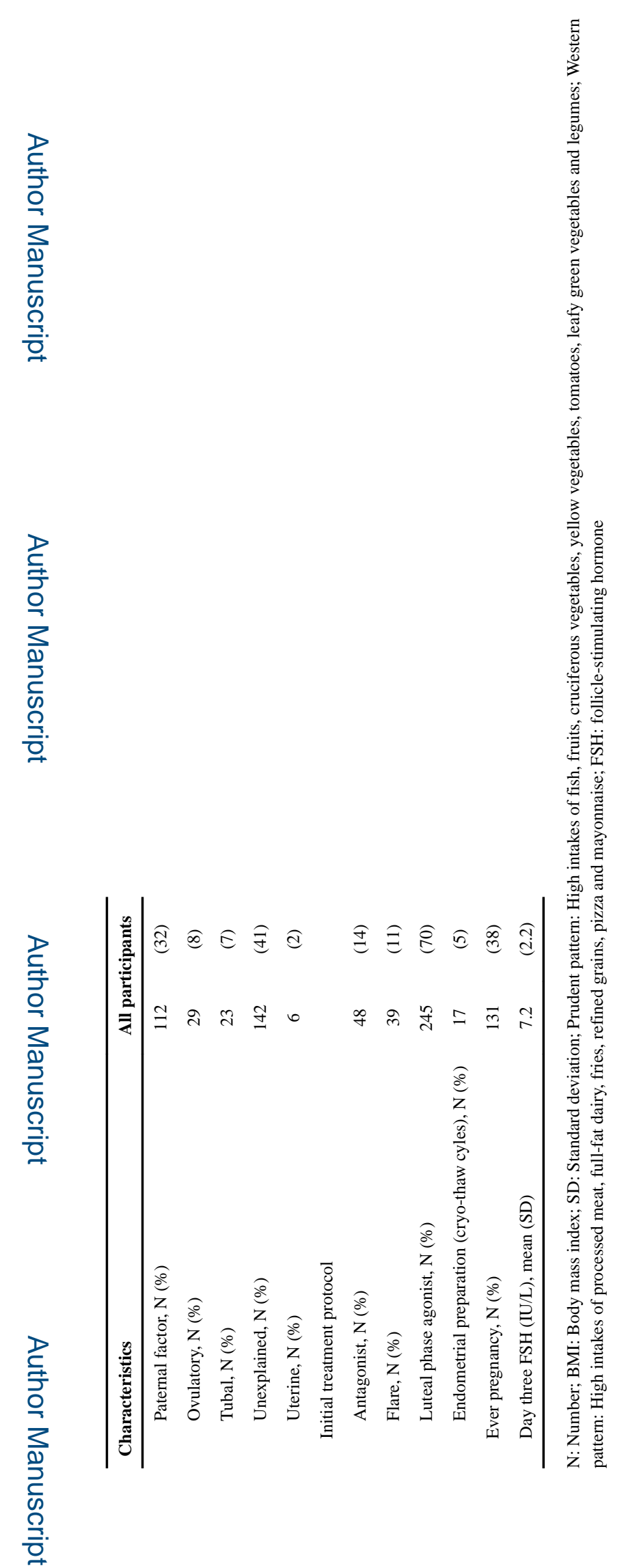

Epidemiology. Author manuscript; available in PMC 2020 May 01. 


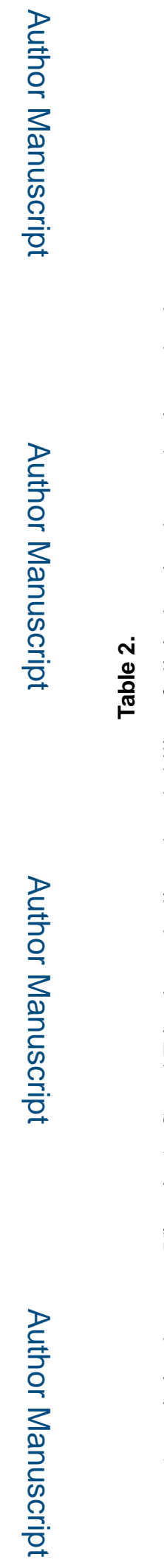

$\mathscr{\Xi}$
0
0
0

.0
00
0
0
0
0
0
0
0
0
0
0
0

䆤| $\mid$ 
I

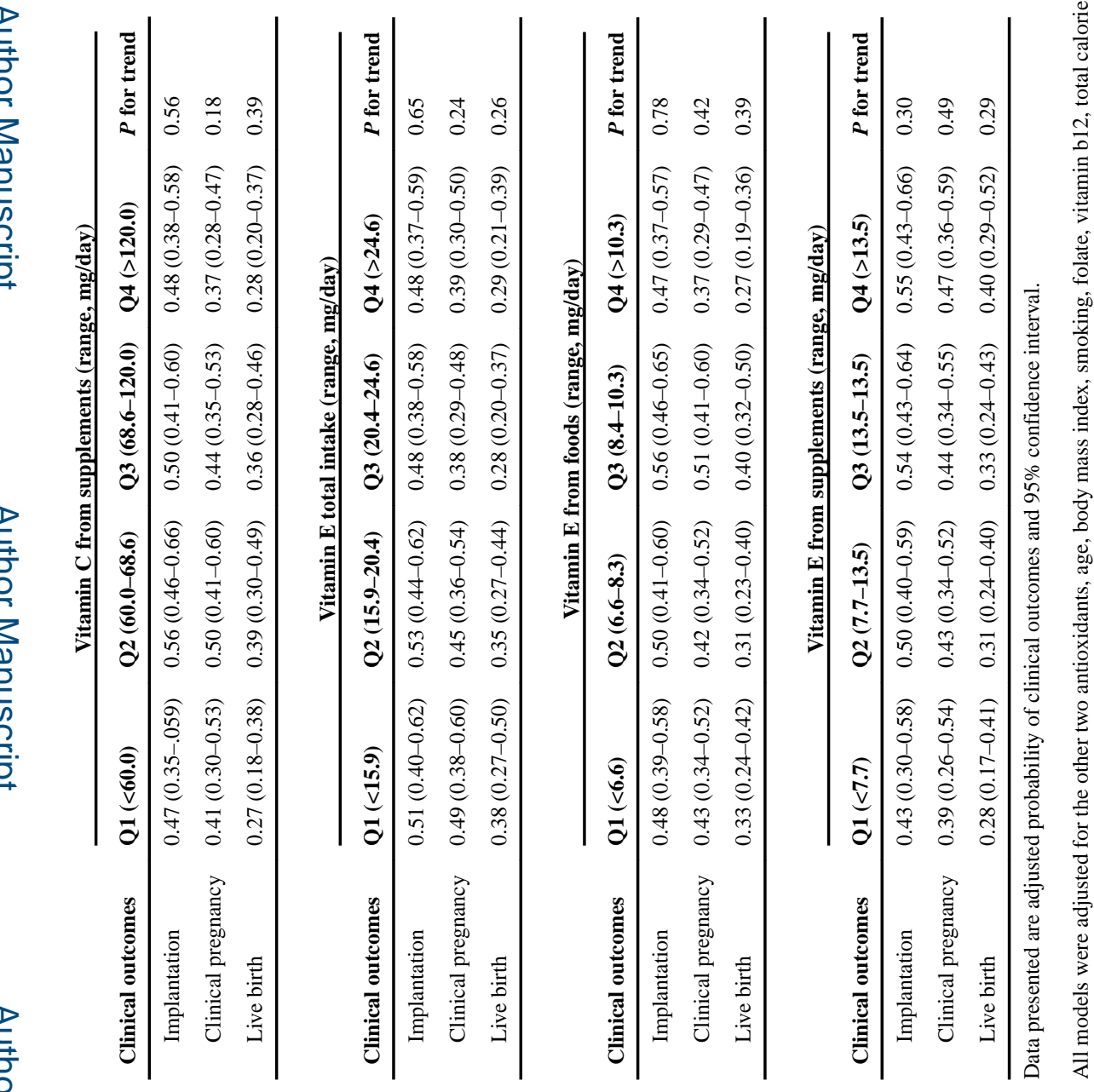



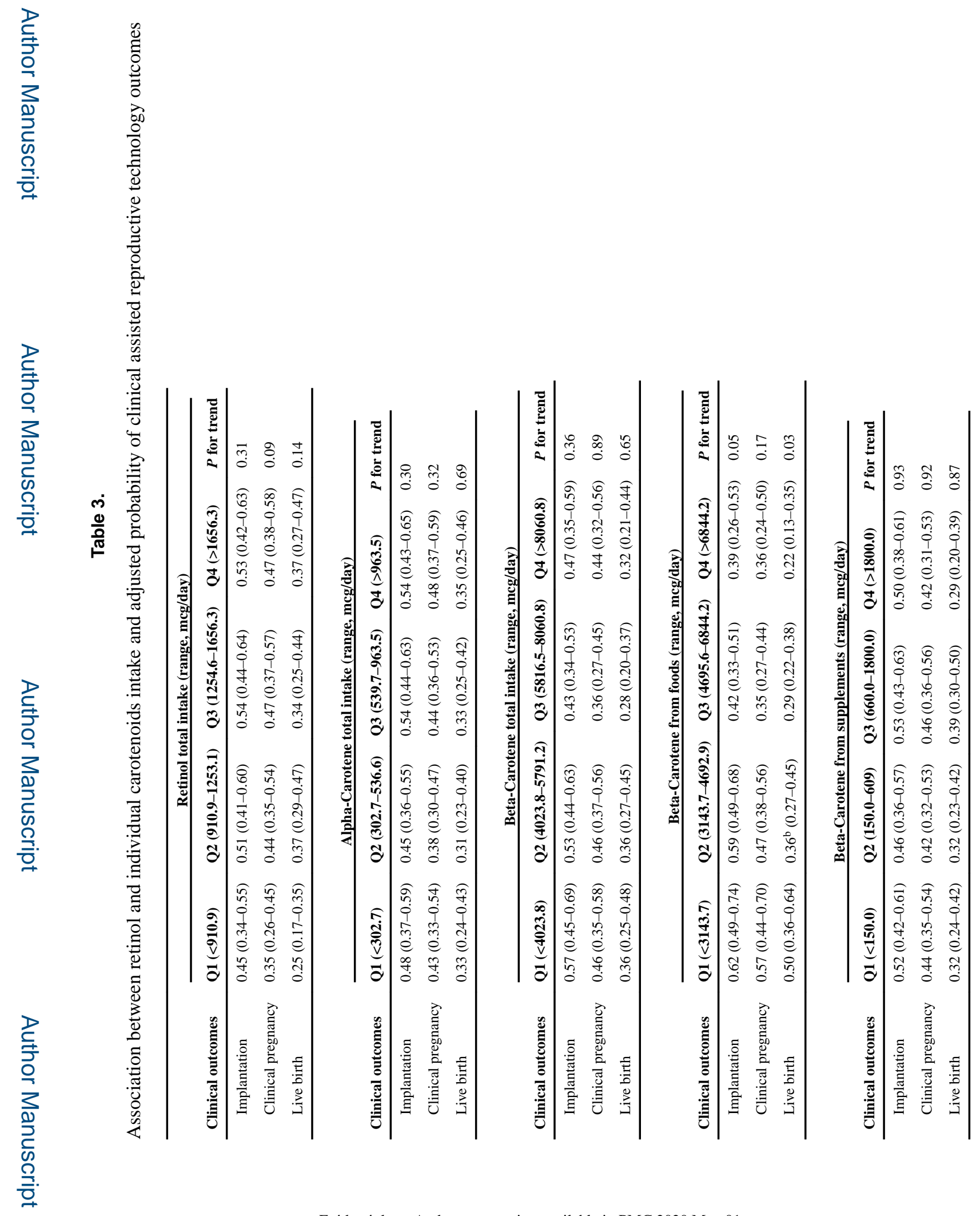

Epidemiology. Author manuscript; available in PMC 2020 May 01. 


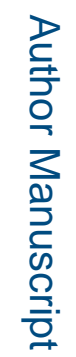

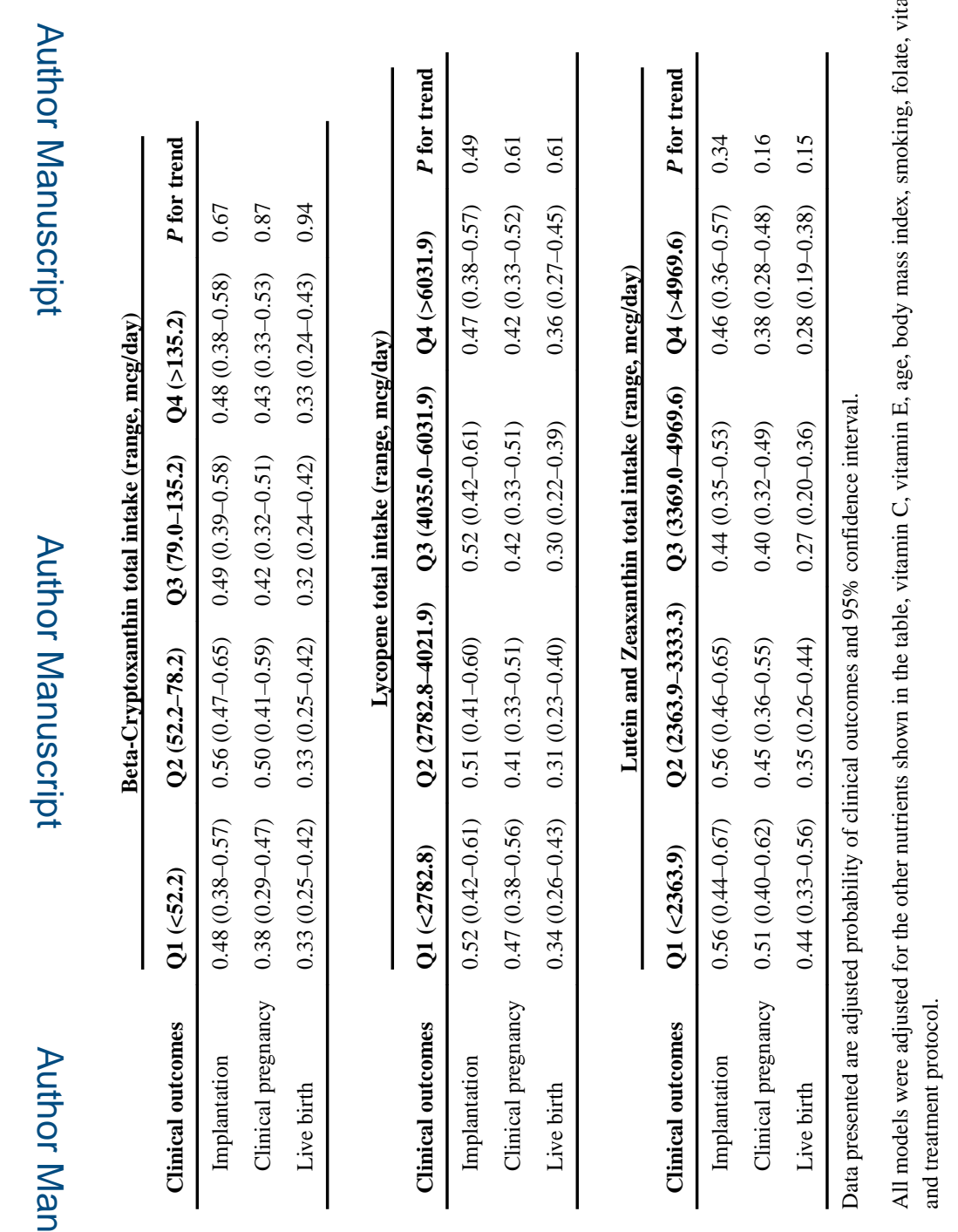

